\title{
Is Advancing Circadian Rhythm the Mechanism of Antidepressants?
}

\author{
Heon-Jeong Lee ${ }^{\bowtie}$ \\ Department of Psychiatry and Chronobiology Institute, Korea University College of Medicine, Seoul, Republic of Korea
}

\begin{abstract}
Antidepressants usually require 2-8 weeks after drug administration to obtain a clinical response. In contrast, three fast-acting antidepressant treatments (sleep deprivation, electroconvulsive therapy, and ketamine) significantly reduced depressive symptoms within hours to days in a subgroup of patients with depressive disorder. This review addresses the mechanisms underlying these fast effects, with specific focus on treatment effects on circadian rhythms. Numerous recent studies have shown that circadian dysregulation may play an important role in the pathogenesis of mood disorders. These studies indicate that a common therapeutic mechanism underlying the three fast antidepressant therapies is related to circadian rhythm. Evidence suggests that depressive disorder is associated with circadian rhythm delay and that the mechanism of the antidepressant effect is a process in which the delayed circadian rhythm is restored to normal by the treatment.

Psychiatry Investig 2019;16(7):479-483
\end{abstract}

Key Words Antidepressant, Circadian, Sleep deprivation, Electroconvulsive therapy, Ketamine.

\section{INTRODUCTION}

The mechanisms of antidepressant treatment have not yet been elucidated. Antidepressant treatment is successful in only approximately $60 \%$ of cases, and even if the same antidepressant is administered to a patient, the therapeutic response is not consistent among depressive episodes, raising questions about the therapeutic mechanism. In addition, synaptic monoamine increases, which are a putative mechanism of antidepressant treatment, occur a few hours after administration of an antidepressant, but it takes from 2-8 weeks for the antidepressant effect to occur. ${ }^{1}$ Thus, the mechanism of antidepressant treatment is not explained by the increased neurotransmitter alone. To address the delayed antidepressant effect, it has been suggested that downregulation of the somatodendritic monoamine autoreceptor, rather than the monoamine concentration itself, is a key mechanism underlying antidepressant efficacy. ${ }^{2}$ Although the delayed response to antidepressant treatment has been described as a window of op-

Received: 10 June, 2019 Accepted: 20 June, 2019

$\triangle$ Correspondence: Heon-Jeong Lee, MD, PhD

Department of Psychiatry, Korea University College of Medicine, 73 Inchonro, Seongbuk-gu, Seoul 02841, Republic of Korea

Tel: +82-2-920-5815, Fax: +82-2-929-7679, E-mail: leehjeong@korea.ac.kr

(a) This is an Open Access article distributed under the terms of the Creative Commons Attribution Non-Commercial License (https://creativecommons.org/licenses/bync/4.0) which permits unrestricted non-commercial use, distribution, and reproduction in any medium, provided the original work is properly cited. portunity for receptor downregulation and neuroplasticity of brain structures, such as the hippocampus and prefrontal cortex, some observations offer new insights into the mechanisms of antidepressant action. These observations pertain to the efficacy of several treatment modalities that are much faster than other antidepressants: sleep deprivation, electroconvulsive therapy, and low-dose ketamine therapy.

\section{FAST BUT TRANSIENT: KEYS TO SOLVE THE ENIGMA}

There is growing evidence that a common therapeutic mechanism underlying the three fast-acting treatments is related to circadian rhythm. Evidence suggests that depressive disorder is associated with circadian rhythm and antidepressanteffect mechanisms are processes by which delayed circadian rhythm is returned to normal by the treatment. This may explain the mechanism of action of fast-acting anti-depressant therapies such as sleep deprivation, electroconvulsive therapy, and low dose ketamine treatment, all of which display an antidepressant effect in several hours to a few days. These three treatment methods have the commonality that they have a rapid, but short-term. Why does this happen? Recent studies have focused on circadian-rhythm normalization (advancing a delayed circadian rhythm) as a common feature of these therapies. 


\section{Sleep deprivation}

People with poor sleep can experience excessive daytime sleepiness, chronic fatigue, anxiety, cognitive dysfunction, and poor motor function. However, sleep deprivation in depressed patients can improve mood faster than any other treatment. Several mechanisms have been hypothesized to explain antidepressant effects associated with sleep deprivation. ${ }^{3-5}$ Restoration of the circadian rhythm is at the forefront of the proposed mechanisms. Patients with major depression have circadian-rhythm abnormalities that aggravates their depression. Failure to normalize the circadian rhythm can lead to longterm consequences that are detrimental to physical and mental health. Hines et al. ${ }^{6}$ suggest that increased glial signaling of adenosine (A1) receptors is largely responsible for the mood enhancement derived from sleep deprivation. Glial cells facilitate increased activation of synaptic $\mathrm{A} 1$ receptors, and the drug 2-chloro-N6-cyclopentyladenosine, an A1 adenosine receptor agonist, improves mood significantly. ${ }^{6}$

Retinal photoreceptors entrain the circadian rhythm to the solar day. The photic resetting involves cAMP response element-binding protein (CREB)-mediated upregulation of Per genes within individual cells of the suprachiasmatic nuclei (SCN). By analyzing the light-regulated transcriptome of the SCN, Jagannath et al. ${ }^{7}$ identified a key role of salt-inducible kinase 1 (SIK1) and CREB-regulated transcription coactivator 1 (CRTC1) in clock re-setting. An entrainment stimulus causes CRTC1 to coactivate CREB, inducing the expression of Per1 and Sik1. SIK1 then inhibits further shifts of the clock by phosphorylation and deactivation of CRTC1. Knockdown of Sik1 within the SCN results in increased circadian phase shifts and rapid re-entrainment to light. Thus, SIK1 provides negative feedback, acting to suppress the effects of light on the internal clock. The increased adenosine generated by sleep deprivation induces inhibition of Sik1 in SCN neurons. The decreased activity of Sik1 leads to the rapid entrainment to light; that is, rapid circadian advancement to re-alignment. This rapid circadian re-alignment could be the mechanism by which rapid antidepressant effects occur following sleep deprivation (Figure 1).

Sleep deprivation can exert antidepressant effects in humans in less than $24 \mathrm{~h}$. However, it is rarely used clinically because the effect disappears once the subject goes back to sleep.

\section{Electroconvulsive therapy}

Electroconvulsive therapy (ECT) is very effective for some patients with severe or refractory depression. In ECT, electrical stimulation is transmitted to the brain, causing seizures. These seizures help to relieve the symptoms of depression, but the mechanism of action is not well understood yet. Because the response to ECT is rapid, it is often used as a treatment

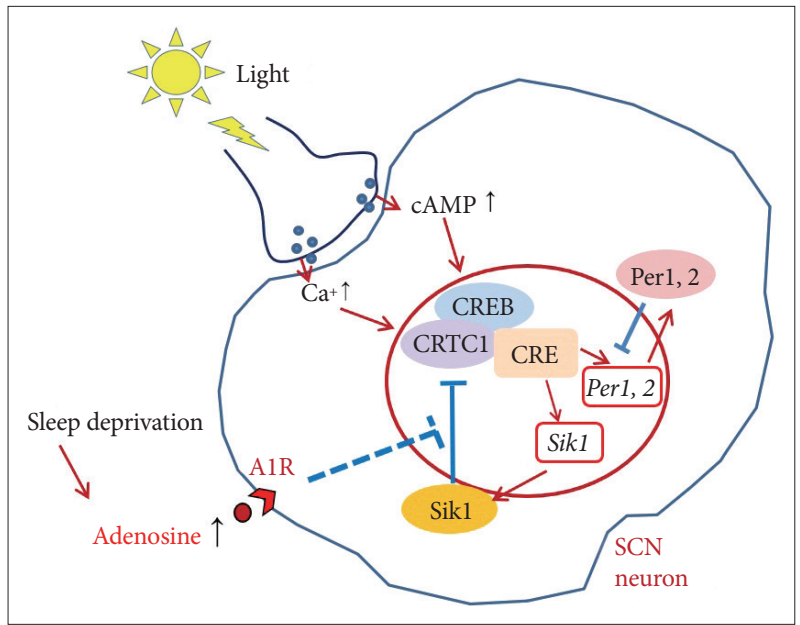

Figure 1. Light from retina entrains the circadian rhythm according to light/dark cycles. The photic reset involves upregulation of Per genes induced by the CREB protein within individual cells of SCNs. By analyzing the light-regulated SCN transcriptome, Jagannath et al. ${ }^{7}$ identified a key role for SIK1 and CRTC1 in the readjustment of the internal clock. An entrainment process causes CRTC1 to coactivate CREB, inducing the expression of Per1, 2 and Sik1. SIK1 then inhibits the subsequent shifts of the clock by phosphorylation and deactivation of CRTC1. Sleep deprivation induces an increase in adenosine signaling in A1R, which inhibits Sik1 in the SCN, and may advance the rapid re-entrainment of circadian rhythm by advancing the delayed rhythm that is present in the clinically depressed state. A1R: adenosine A1 receptor, CRE: CAMP responsive element, CREB: CRE binding, CRTC1: CREB1-regulated transcription coactivator, SCNs: suprachiasmatic nuclei, SIK1: salt-inducible kinase 1.

for people with severe psychotic or suicidal depression. For these people, waiting for antidepressants or other treatments to take effect is highly undesirable. However, the drawback of ECT is that its effects usually do not persist, and additional or other treatments are usually needed. ${ }^{8}$

Although the exact mechanisms of action of ECT have not yet been elucidated, several hypotheses have been suggested. Among these hypotheses, neuroendocrine theory, proposed by Fink and Ottosson, ${ }^{9}$ postulates that the antidepressant effect of ECT is due to the persistent release of hypothalamic substances that induce mood changes from depression to normal mood states, with concomitant modification of vegetative functions and improvement of disturbed sleep patterns. It is also assumed that ECT increases neurotransmission in general, normalizes neuroendocrine functioning, and increases neuronal and synaptic growth, leading to improved brain function in general and cognitive functioning, sleep, and mood in particular., ${ }^{9,10}$

In a study using actigraphy to determine the potential acute effects of ECT on sleep parameters, researchers found no difference in sleep quality between nights after ECT and other nights, neither in subjective nor objective parameters of the evaluation of actigraphy. ${ }^{11}$

Circadian-rhythm normalization has been regarded as a 
possible mechanism underlying the antidepressant effect of ECT. Szuba et al. ${ }^{12}$ reported that elevated body temperature in subjects with depression fell to normal following recovery after electroconvulsive therapy, suggesting that elevated body temperature is a state-specific correlate of depression. The 24hour profile of temperature was significantly different in patients pre-ECT than in patients post-ECT or in controls. PostECT subjects and controls exhibited similar 24-hour temperature profiles. That is, ECT normalizes the timing and increases the amplitude of circadian temperature rhythm in patients with depression. ${ }^{12}$

Kim et al. ${ }^{13}$ demonstrated that electroconvulsive seizure (ECS) alters the expression and daily oscillation of circadian genes in the frontal cortex of rats. Specifically, circadian rhythms of expression were generally advanced after repeated ECS. However, their study was conducted in normal animals, not in animal models of depression with disturbances in circadian rhythm. ${ }^{13}$ Therefore, it is difficult to confirm that delayed circadian rhythm in depression is advanced by ECS. In another study of patients with major depressive disorder who did not respond to pharmacological treatment, ECT resulted in a significant decrease in symptoms of depression and urinary excretion of 6-sulfatoxymelatonin, a metabolite of melatonin. ${ }^{14}$ ECT often generated rapid initial antidepressant effects. Although these effects rapidly disappear, they gradually build over a full course of ECT, which leads to a more sustained antidepressant effect.

\section{Ketamine}

The N-methyl-D-aspartate receptor (NMDAR) antagonist ketamine has attracted considerable interest in mental health research because of its rapid antidepressant action; however, its mechanism of action has remained elusive. Li et al..$^{15}$ examined 12,000 transcripts and found that key clock genes, essential for controlling virtually all body rhythms, had robust 24-hour sinusoidal expression patterns in six brain regions in control subjects. In patients with MDD, expression patterns of clock genes in the brain were significantly dysregulated when matched for time of day with controls. Some of the most robust changes have been observed in the anterior cingulate cortex (ACC). Structural abnormalities, lesion studies, and a large number of functional brain imaging studies indicate increased activation of the ACC in patients with depression, and there may be circadian dysregulation of clock gene expression in a subgroup of patients with MDD. Data from human, animal, and neuronal cell studies suggest that low-dose ketamine and sleep deprivation can modulate circadian rhythms. ${ }^{16}$ These findings suggest that the rapid antidepressant actions of ketamine and SD may, in part, reset abnormal clock genes in MDD to restore and stabilize circadian rhythms. Conversely, clinical relapse may reflect desynchronization of the clock, indicating reactivation of an abnormal function of the clock gene.

Duncan et al. ${ }^{17}$ examined the rapid antidepressant effects of ketamine on markers of circadian rhythm (amplitude and timing) in mood disorders using wrist actigraphy. The amplitude and timing of circadian activity at baseline, day 1 , and day 3 after infusion of ketamine were measured in 51 patients with MDD or bipolar disorder. Compared with placebo and baseline, mood-independent decrease of the mesor was present on D1 after ketamine treatment. Mood-related circadian effects differed between fast responders and non-responders at day 1 and 3. At baseline, an advanced circadian phase and a lower-level of activity distinguished subsequent responders from non-responders. On day 1, non-responders to ketamine had a lower mesor and a blunted 24-hour amplitude compared to baseline. The difference in activity level between responders and non-responders at baseline reversed by day 3, with $17 \%$ higher activity levels in responders than in non-responders. Responders had a greater amplitude and more activity than non-responders. The results revealed a link between the clinical antidepressant effects of ketamine and the circadian rhythm. The results suggest that trait-like circadian activity patterns are markers for a rapid mood-response to ketamine, and that mediators of continuing ketamine-induced mood changes include altered timing and amplitude of the circadian system. ${ }^{17,18}$

Orozco-Solis et al. ${ }^{19}$ compared the effect of ketamine treatment and $\mathrm{SD}$ on gene transcription in the anterior cingulate cortex (the focus of circadian dysfunction in depressive disorders) in mice. In addition to demonstrating similar antidepressant effects of both interventions via the forced swimming test, they identified 64 genes whose expression is influenced by both ketamine and SD. Using the Ontology Gene Database and the Encyclopedia of Kyoto Genes and Genomes, these genes were ranked according to molecular function and interrogated to determine under-representation or overrepresentation of particular functional groups. The results of this analysis revealed genes involved in neuronal plasticity, synaptic transmission, neurological development, and cell signaling. Enrichment analysis of gene sets was used to identify the biological functions associated with the genes. This highlighted the role of circadian rhythm, with five transcripts of circadian genes downregulated by both ketamine and SD. The researchers hypothesized that ketamine affects circadian gene transcription through its inhibitory actions on the CLOCK/ BMAL1 heterodimer (which itself drives clock-gene transcription), and that sleep deprivation may work similarly. This putative mechanism for a circadian gene reset is also a mechanism that may, at least in part, explain the rapid antidepressant effects of ketamine.

Recently, Yang et al. ${ }^{20}$ showed that the blockade of NMDAR- 
dependent disruption activity in the lateral habebula $(\mathrm{LHb})$ governs the rapid antidepressant actions of ketamine in rat and mouse models of depression. LHb neurons significantly increase burst activity and theta-band synchronization in depressive-like animals, which is reversed by ketamine. The researchers suggested a simple model, whereby ketamine quickly elevates mood by blocking NMDAR-dependent bursting activity of $\mathrm{LHb}$ neurons to disinhibit downstream monoaminergic reward centers, thereby providing a framework for developing new rapid-acting antidepressants. However, there is an alternative interpretation of this result. The main circadian clock in mammals is located in the hypothalamic suprachiasmatic nucleus (SCN), but central timing mechanisms are also present in brain structures other than the SCN. LHb is known for its important role in regulation of the monoaminergic system. Since monoaminergic systems show circadian rhythm, it is possible that the role of the LHb clock is to modulate the rhythmic activity of the dopamine, serotonin, and norepinephrine systems and associated behaviors. Thus, disturbances of circadian activity in LHb could, in part, cause these rhythmic alterations in mood disorders. ${ }^{21,22}$ Given the role of LHb, NMDAR-dependent LHb-blocking neuronal activity that leads to disinhibition of downstream monoaminergic centers can be interpreted differently: The blocking activity of ketamine in the LHb neuron may induce the resetting of circadian misalignment to circadian normality.

\section{WHY DOES DEPRESSION RAPIDLY RELAPSE?}

Why do depressive episodes relapse after a fast, transient treatment response? Evidence is accumulating for an association between circadian misalignment and disordered mood. Delayed circadian phase may be a core pathophysiological mechanism underlying depressive episodes. My group suggests that acute mood episodes of mood disorders are related to circadian misalignment between the individual's endogenous circadian rhythms and the individual's physical environment. We have found that acute manic episodes are usually associated with biochemical circadian rhythm acrophases averaging 7 hours more advanced (earlier) than those of controls, although these acrophases could also result from a 17 hour clockwise delay. Mixed manias are phase-delayed by 6 hours, whereas depression is associated with $4-5$ hour phase delays compared to controls. The phase shift in the circadian rhythm is normalized before the patient is discharged. The length of hospital stay varies from two weeks to three months; normalization of circadian rhythm requires time. ${ }^{23}$

The circadian clock is self-sustained or endogenous-circadian rhythms persist even in the absence of environmental inputs. The circadian rhythm is entrainable by external zeitgebers, but this is generally difficult, and needs considerable time. In particular, even though the transient circadian rhythm may be advanced in a certain brain region by fast-acting antidepressants, the treatment effect is not sustained unless the individual's endogenous circadian rhythm is completely normalized.

Because the most powerful zeitgeber is light, it is very important to retain regular life patterns that follow a day/night cycle in order to restore normal circadian rhythm. Light therapy for normalizing circadian misalignment is also important, especially in depression during winter. ${ }^{24}$

\section{Acknowledgments}

This study was supported by the Korea Health 21 R\&D Project funded by the National Research Foundation of Korea (2017M3A9F1031220).

\section{Conflicts of Interest}

The author has no potential conflicts of interest to disclose.

\section{ORCID iD}

Heon-Jeong Lee https://orcid.org/0000-0002-9560-2383

\section{REFERENCES}

1. Stassen HH, Delini-Stula A, Angst J. Time course of improvement under antidepressant treatment: a survival-analytical approach. Eur Neuropsychopharmacol 1993;3:127-135.

2. Stahl SM. Stahl's Essential Psychopharmacology: Neuroscientific Basis and Practical Applications. 4th Edition. Cambridge, MA: Cambridge University Press; 2013.

3. Goldstein MR, Plante DT, Hulse BK, Sarasso S, Landsness EC, Tononi $\mathrm{G}$, et al. Overnight changes in waking auditory evoked potential amplitude reflect altered sleep homeostasis in major depression. Acta Psychiatr Scand 2011;125:468-477.

4. Landsness EC, Ferrarelli F, Sarasso S, Goldstein MR, Riedner BA, Cirelli $\mathrm{C}$, et al. Electrophysiological traces of visuomotor learning and their renormalization after sleep. Clin Neurophysiol 2011;122:2418-2425.

5. Hanlon EC, Vyazovskiy VV, Faraguna U, Tononi G, Cirelli C. Synaptic potentiation and sleep need: clues from molecular and electrophysiological studies. Curr Top Med Chem 2011;11:2472-2482.

6. Hines DJ, Schmitt LI, Hines RM, Moss SJ, Haydon PG. Antidepressant effects of sleep deprivation require astrocyte-dependent adenosine mediated signaling. Transl Psychiatry 2013;3:e212.

7. Jagannath A, Butler R, Godinho SIH, Couch Y, Brown LA, Vasudevan SR, et al. The CRTC1-SIK1 pathway regulates entrainment of the circadian clock. Cell 2013;154:1100-1111.

8. Brakemeier EL, Merkl A, Wilbertz G, Quante A, Regen F, Bührsch N, et al. Cognitive-behavioral therapy as continuation treatment to sustain response after electroconvulsive therapy in depression: a randomized controlled trial. Biol Psychiatry 2014;76:194-202.

9. Fink M, Ottosson JO. A theory of convulsive therapy in endogenous depression: significance of hypothalamic functions. Psychiatry Res 1980;2:49-61.

10. Bolwig TG. How does electroconvulsive therapy work? Theories on its mechanism. Can J Psychiatry 2011;56:13-18.

11. Hoogerhoud A, Hazewinkel AW, Reijntjens RH, van Vliet IM, van Noorden MS, Lammers GJ, et al. Short-term effects of electroconvulsive therapy on subjective and actigraphy-assessed sleep parameters in severely depressed inpatients. Depress Res Treat 2015;2015:764649.

12. Szuba MP, Guze BH, Baxter LR Jr. Electroconvulsive therapy increases 
circadian amplitude and lowers core body temperature in depressed subjects. Biol Psychiatry 1997;42:1130-1137.

13. Kim SH, Park HG, Jeong SH, Kang UG, Ahn YM, Kim YS. Electroconvulsive seizure alters the expression and daily oscillation of circadian genes in the rat frontal cortex. Psychiatry Investig 2018;15:717-726.

14. Krahn LE, Gleber E, Rummans TA, Pileggi TS, Lucas DL, Li H. The effects of electroconvulsive therapy on melatonin. J ECT 2000;16:391398.

15. Li JZ, Bunney BG, Meng F, Hagenauer MH, Walsh DM, Vawter MP, et al. Circadian patterns of gene expression in the human brain and disruption in major depressive disorder. Proc Natl Acad Sci U S A 2013; 110:9950-9955.

16. Bunney BG, Li JZ, Walsh DM, Stein R, Vawter MP, Cartagena P, et al. Circadian dysregulation of clock genes: clues to rapid treatments in major depressive disorder. Mol Psychiatry 2015;20:48-55.

17. Duncan WC Jr, Slonena E, Hejazi NS, Brutsche N, Yu KC, Park L, et al. Motor-activity markers of circadian timekeeping are related to ketamine's rapid antidepressant properties. Biol Psychiatry 2017;82:361369.

18. Duncan WC Jr, Slonena EE, Hejazi NS, Brutsche N, Park LT, Henter
ID, et al. Are 24-hour motor activity patterns associated with continued rapid response to ketamine? Neuropsychiatr Dis Treat 2018;14: 2739-2748.

19. Orozco-Solis R, Montellier E, Aguilar-Arnal L, Sato S, Vawter MP, Bunney BG, et al. A circadian genomic signature common to ketamine and sleep deprivation in the anterior cingulate cortex. Biol Psychiatry 2017;82:351-360.

20. Yang Y, Cui Y, Sang K, Dong Y, Ni Z, Ma S, et al. Ketamine blocks bursting in the lateral habenula to rapidly relieve depression. Nature 2018;554:317-322.

21. Mendoza J. Circadian neurons in the lateral habenula: clocking motivated behaviors. Pharmacol Biochem Behav 2017;162:55-61.

22. Baño-Otálora B, Piggins HD. Contributions of the lateral habenula to circadian timekeeping. Pharmacol Biochem Behav 2017;162:46-54.

23. Moon JH, Cho CH, Son GH, Geum D, Chung S, Kim H, et al. Advanced circadian phase in mania and delayed circadian phase in mixed mania and depression returned to normal after treatment of bipolar disorder. EBioMedicine 2016;11:285-295.

24. Choukroun J, Geoffroy PA. Light therapy in mood disorders: a brief history with physiological insights. Chronobiol Med 2019;1:3-8. 